LA-UR-96:-

$99-129$

$$
\text { CONF-981211- }
$$

Los Alamos National Laboratory is operated by the University of California for the United States Department of Energy under contract W-7405-ENG-36

TITLE: An Approach to Fundamental Study of Beam Loss Minimization

AUTHOR(S): R.A. Jameson, LANL

SUBMTTED TO:

Proceedings of Workshop on Space Charge Dominated Beam Physics for

Heavy Ion Fusion, 10-12 December 1998, Institute of Physical and Chemical Research (RIKEN),

Wako-shi, Japan

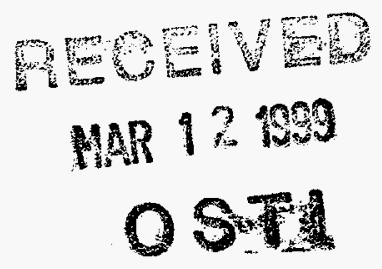

By acceptance of this article, the publisher recognizes that the U.S. Government retains a nonexclusive, royalty-free license to publish or reproduce the published form of this contribution, or to allow others to do so, for U.S. Government purposes.

The Los Alamos National Laboratory requests that the putilisher identify this article as work performed under the auspices ofthe U.S. Department of Energy.

LOS ADImOS

Los Alamos National Laboratory Los Alamos, New Mexico 87545

FORM NO. $836 \mathrm{R4}$ ST NO 2629 5/B1 


\section{DISCLAIMER}

This report was prepared as an account of work sponsored by an agency of the United States Government Neither the United States Governmeat aor any agency thereof, nor any of their empioyees, makes any warranty, express or implied, or assumes any legal liability or responsibility for the accuracy, compieteness, or usefulness of any information, apparatus, product, or process disctosed, or represents that its use would not infringe privately owned rights. Refereace berein to any speciric commercial product, process, or service by trade name, trademark, inanufacturer, or otherwise does not necessarily constitute or imply its endorsement, recommendation, or favoring by the United States Governmeat or any agency thereof. The views and opinions of authors expressed berein do not necessarily state or refiect those of the United States Government or any agency thereof. 


\section{DISCLAIMER}

Portions of this document may be illegible in electronic image products. Images are produced from the best available original document. 


\title{
An Approach to Fundamental Study of Beam Loss Minimization
}

\author{
R.A. Jameson \\ LANSCE-1 MS H808 \\ Los Alamos National Laboratory \\ Los Alamos, New Mexico, 87545 USA
}

\begin{abstract}
The accelerator design rules involving rms matching, developed at CERN in the 1970 's, are discussed. An additional rule, for equipartitioning the beam energy among its degrees of freedom, may be added to insure an rms equilibrium condition. If the strong stochasticity threshold is avoided, as it is in realistic accelerator designs, the dynamics is characterized by extremely long transient settling times, making the role of equipartitioning hard to explain. An approach to systematic study using the RFQ accelerator as a simulation testbed is discussed. New methods are available from recent advances in research on complexity, nonlinear dynamics, and chaos.
\end{abstract}

\section{RMS MATCHING}

The top-level specifications for high-intensity particle accelerators include high availability ${ }^{1,2}$. A major availability factor is beam loss minimization, to insure maintainability without remote manipulators ${ }^{34}$, .

There is not much design guidance for beam loss minimization, especially as allowed beam losses are very low, of order $1 \mathrm{nA} / \mathrm{m} / \mathrm{GeV}$ for rf light ion linacs; e.g., 1 part in $10^{8} / \mathrm{m} / \mathrm{GeV}$ for a $100 \mathrm{~mA}$ average current proton linac, resolution beyond the fidelity of existing simulations. The traditional rules are that the beam must be rms matched in the transverse and longitudinal planes:

$$
\varepsilon_{t n}^{2}=\frac{a^{4} \gamma^{2} \sigma^{t^{2}}}{n^{2} \lambda^{2}}=\frac{a^{4} \gamma^{2}}{n^{2} \lambda^{2}}\left(\sigma_{o}^{t^{2}}-\frac{I \lambda^{3} k(1-f f)}{a^{2}(\gamma b) \gamma^{2}}\right)
$$




$$
\varepsilon_{\ln }^{2}=\frac{(\not b)^{4} \gamma^{2} \sigma^{t^{2}}}{n^{2} \lambda^{2}}=\frac{(\not b)^{4} \gamma^{2}}{n^{2} \lambda^{2}}\left(\sigma_{o}^{t^{2}}-\frac{2 I \lambda^{3} k f f}{a^{2}(\not b) \gamma^{2}}\right)
$$

where $t$ and $l$ denote transverse and longitudinal, $\varepsilon$ is rms emittance, $a$ and $b$ are transverse and longitudinal rms beam radii (assuming an ellipsoidal distribution), $\sigma$ is a phase advance with beam current, $\sigma_{o}$ is the external field phase advance, $\lambda$ is $\mathrm{rf}$ wavelength, $n$ is the number of $\beta \lambda 2$ in a transverse focusing period, $I$ is the beam current, $f f$ is the geometry factor $\approx a / 3 b, \gamma$ and $\beta$ are the relativistic gamma and beta, and $k=\frac{3 n^{2}}{8 \Pi} \frac{z_{0} q 10^{-6}}{m c^{2}}$. All but two of the parameters must be specified by other means.

The matching equations insure that emittance is not diluted by betatron or synchrotron oscillations. They provide obvious guidance on what to do when there is a transition in the machine of any kind that affects the phase advance: as beam emittance and size would be kept constant across the transition, the focusing per unit length must also be kept constant across the transition. The rms matching equations also guide one in the direction of higher frequency to maximize beam brightness, the ratio of beam current to multidimensional beam emittance, as basically less charge is accelerated per bucket, reducing the space-charge term. However, brightness, characterizing the beam core, is not the same as residual beam loss, which comes from scraping off particles in a tenuous cloud outside the core:

\section{HALO DIAGNOSTICS}

Practical designs are done using detailed multiparticle simulation codes to observe what happens along a machine, including the effects of various errors. Observed beam boundaries are multiplied by experience-based engineering safety factors to arrive at adequate beam tube dimensions and focusing strengths.

Recently, a great deal has been learned about one common form of practical error that of $\mathrm{rms}$ mismatch[4], which drives parametric resonances. This particular type of error is bounded because a particle's tune is a function of radius. Thus most investigations have concentrated on approximating the boundaries, using a simple model of a stably oscillating core field to influence the motion of test particles arbitrarily initialized ${ }^{5}$. Such a boundary estimation, useful for setting engineering safety factors, emphasizes what might happen, if the beam core remains as simple as the model describes, and if particles that describe the larger mismatch orbits could actually reach the appropriate initial conditions. 
If one is interested in probing deeper into the mechanisms that can drive particles into unwanted areas of phase-space (e.g., beyond the bore, or into highly nonlinear parts of the rf bucket), it is necessary to go beyond observing what happens and ask how, why, when, where, which, etc.

In this case, very simplified models and test particle methods are severely limited. The idealized core/test-particle model for rms mismatch, for example, contains no information about particle transport, and can give no information about how, why, which, when, where particles might reach a point in phase-space from which they would make the larger mismatch orbits.

These questions must usually be explored using detailed diagnostics on a fully selfconsistent particle simulation code. "Self-consistent" means that all the particles are involved in both producing the field and being influenced by the field. Typically, both the rms characteristics of the beam as well as the particle orbits are functions of time, and this makes simple periodic measurements, such as the usual Poincarè plots, problematic. Methods for observing transport and beam halo are detailed in [4] for a mismatched beam in a self-consistent $\left(x, x^{\prime}, y, y^{\prime}\right)$ simulation, symmetrized to $\left(r, r^{\prime}\right)$, in a continuous focusing channel. Particle transport is seen to involve resonance entraining and hopping. Particle escape from the core into the mismatch halo can be precisely defined ${ }^{\mathrm{a}}$ by defining a local particle tune and noting when the particle's tune accumulates to $2 \Pi$ in the period of the resonance under observation. This allows very detailed information to be obtained concerning whether particles are in the core or in the halo, when a particle enters or leaves the halo, how long it stays in the halo, and so on.

The single-particle local instantaneous tune in [4] is simply based on the local force balance on the particle, i.e., the local restoring force minus the space-charge force on the particle from all particles inside the particle's physical orbit radius.

Advantages of this method are that it is easy to compute, and rigorously replaces the laborious methods of resonance overlap and turnstile computation that previously were used in the study of nonlinear dynamics and chaos ${ }^{6}$.

\section{EQUIPARTITIONING}

The two matching equations alone are insufficient to prevent emittance growth of an intense beam as space-charge forces begin to dominate over the emittance terms. For example, steady longitudinal rms emittance growth is seen in conventional designs

" Most "definitions" of halo are very fuzzy, and there is no consensus. 
with fixed accelerating field gradient (which gives decreasing longitudinal focusing with energy) and constant transverse focusing. Also, although rms transverse emittance growth can be kept very small in such designs, the total effective transverse (and longitudinal) emittance growth is large. It would be desirable to add other rules based on space-charge physics to prevent this emittance growth and other sources of potential beam loss.

Only one additional possible rule is presently known - the rule of rms equipartitioning (EP) ${ }^{7}$.

An equipartitioned beam bunch has equal energy in the transverse and longitudinal directions, leaving no free, unbalanced energy that could cause emittance growth via coupling mechanisms. A fully matched and equipartitioned bunch would balance out free energy and be matched to all orders, i.e., a truly stationary, equilibrium distribution. Such a distribution is beyond analysis, but such distributions do tend to form in actual accelerator channels. For design purposes, we can place an energy balance condition on the rms beam properties:

$$
\frac{e_{\mathrm{ln}} \sigma^{l}}{e_{t n} \sigma^{t}}=1
$$

Locally solving Eq. (3) simultaneously with (1) and (2) yields linac designs that are equipartitioned throughout. Addition of the equipartitioning condition puts a strong constraint on the design space; for example, if $\varepsilon_{l n} / \varepsilon_{t n}=2$ throughout, then $\sigma^{d} / \sigma^{d}=1 / 2$ throughout.

Thresholds for the low-order coherent instabilities of KV beams have been derived, and shown to be closely related to corresponding instabilities for realistic beam distributions as well ${ }^{7,8}$. In reference [3], it is shown that the EP design trajectory is in a region of tune space that is free of low-order instabilities down to quite large tune depressions, and how the EP condition might be chosen in practical cases.

Simulations of equipartitioned channels show characteristics expected of an equilibrium distribution. For example, consider a channel again having constant accelerating gradient. The transverse focusing is then weakened along the channel to achieve EP. The longitudinal rms emittance growth is no longer seen, and the beam distribution remains "tight", with nearly constant ratio between total and rms beam size (or emittance). The improved longitudinal emittance is important for storage ring injection and has led to acceptance of EP designs for this purpose. 
Despite this important evidence, and even acceptance for storage ring injection, almost no further systematic work on this fundamental possibility to achieve an equilibrium beam condition, at least to rms order, has been supported to date.

To some extent, this may be because there are questions about whether EP can provide an equilibrium sufficiently advantageous, for example with respect to total beam size or reduced sensitivity to errors.

One question involves beam size in designs with constant accelerating gradient, an economic constraint in room-temperature linacs. As noted above, this means that the longitudinal focusing decreases with energy. With constant transverse focusing, rms transverse beam size decreases, but total transverse beam size increases considerably. If the transverse focusing is weakened corresponding to the longitudinal focusing to produce EP, the rms transverse beam size naturally grows along the structure. This might seem counterintuitive to beam loss minimization, but total, not rms, beam size is what is important. After acceleration to order $1 \mathrm{GeV}$, the total beam size for both designs appears, from simulations, to be "roughly similar". Looking only at the output ignores the possibility for lower beam losses all along the structure with the tighter beam distribution of the EP design, compared to the more diffuse distribution with tenuous halo characteristic of the constant focusing design. Systematic studies are needed, including error sensitivity studies.

Another reason is fundamental to the accelerator dynamics in the typical design tune space, whether or not equipartitioned.

It has long been observed that a matched beam with energy unbalance of up to around $50 \%$ can be injected "without much effect" on the emittances. Also, if a strongly energy-unbalanced (but matched) beam is injected, the distribution will at first move strongly toward equipartition. But as the EP ratio approaches some value in the vicinity of one, the EP ratio will abruptly level off and not proceed further toward one, or may oscillate around one. From the low-order mode charts of Hofmann discussed in [3], this behavior is not inconsistent with the low-order mode thresholds, and freedom from low-order instability in the EP region.

This behavior is quite well explained if one turns to the literature of complexity, nonlinear dynamics, and chaos. Accelerator beam dynamics is a typical nonlinear system, in which stochasticity thresholds appear as a function of the strength of a nonlinearity parameter. If the nonlinearity, e.g. lack of energy balance, is above a strong stochasticity threshold, very strong mixing (chaos, turbulence) occurs and the system is driven toward an equilibrium. Such mixing can be loosely described as a "thermalization" process, and effective settling times can be short. 
When the above system equilibrates such that the nonlinearity strength falls below the strong stochasticity threshold, the dynamics changes fundamentally.

Now the transient settling time becomes very long, approaching infinity. Quasiequilibria, or meta-equilibria, may still exist. In practical terms, for example, in the case of mismatched beams as discussed in [4], the conditions under which particles actually escape from the beam core onto the large mismatch orbits, the time intervals that particles spend in the core or in the halo, etc., are all random (while still a function of the nonlinearity strength). This property accounts for the observation that the exit time for particles to assume mismatch orbits tends to infinity for a mismatch approaching zero. Or, in the case of non-EP'd injection, the distribution approaches the EP condition until the threshold is crossed, whereupon the setting time becomes very long.

Most modern high-intensity linac (and ring) designs, whether equipartitioned or not, tend to lie in regions of tune-space where the nonlinearity strengths are smaller than the strong stochasticity threshold. (Older linacs of LAMPF vintage violate the threshold at low energy, and have strong emittance growth in this region.)

Below the strong stochasticity threshold, it is not proper to characterize the behavior as "thermalization". The beam would be in equilibrium if completely nonlinearly matched and energy balanced, but this equilibrium will not be a "thermal" one.

The characteristics of long settling times, then, makes it fundamentally quite unclear whether, when, how, etc., the EP condition may or may not be of benefit in accelerator design. (It should be pointed out that the time scales for intense, relatively short linacs and less intense but long ring channels are equivalent.)

Many details of this question are being explored outside the accelerator field by researchers in complexity, nonlinear dynamics and chaos, applied to classical mechanics, astrophysics and other areas. Basically, the question is whether the ergodic hypothesis is true below the strong stochasticity threshold.

\section{RFQ TESTBED FOR STUDY OF EQUIPARTITIONING}

Let us accept that an equilibrium design condition, even if only an rms one, is desirable, and discuss how systematic investigation might proceed.

The author's preference is to test the design in a simulation of a real accelerator, rather than constructing a simplified model. The RFQ has been chosen as the testbed. This 
is probably the most complex situation, because the beam is dc at injection and becomes bunched. Difficult as it may be, this is preferred because it forces attention to real factors. The RFQ is also a quite generic linac; for example, when a frequency jump is made between linac sections, the beam becomes long longitudinally and often the external fields would be shaped to shorten the bunch length again. On other ways, the RFQ is a simpler system, in that its fields are easy to describe analytically.

The RFQ generation is performed in steps. First, specifications are given at the end of a shaper (e.g., $\varepsilon_{\mathrm{n}} / \varepsilon_{\mathrm{n}}=2, \phi_{\mathrm{s}}=-88^{\circ}$, aperture $-\beta \lambda / 4$ along with the current, frequency and injection energy), and the modulation and beam dimensions are solved for to satisfy the matching and EP conditions. The injection section from input to the shaper end is then designed according to rules. The beam is to be equipartitioned from the shaper end through the rest of the RFQ; at each step (typically $1 / 10$ th of a cell), all parameters except the modulation and beam dimensions are found by solving the matching and EP equations simultaneously and exactly. (To date, machine layouts by others have approached the EP condition only approximately by trial and error, which compromises interpretation of the results.)

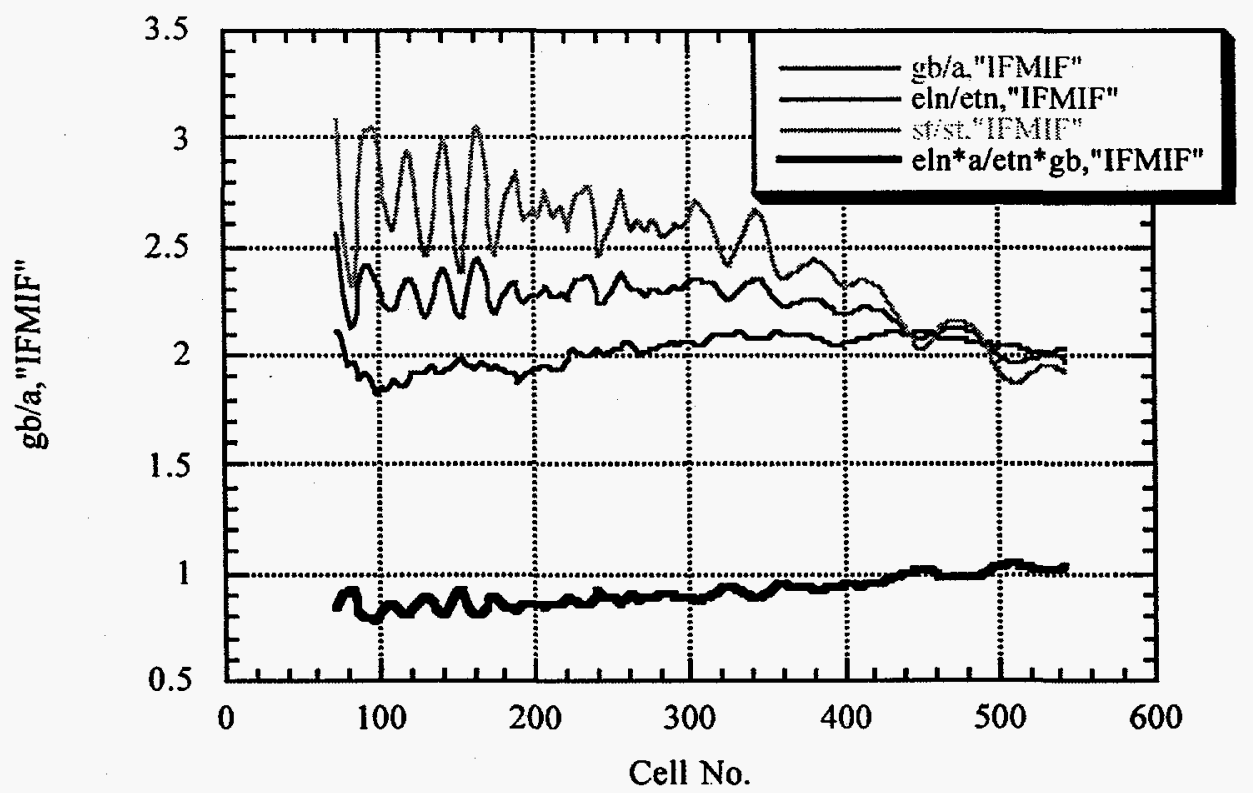

FIGURE 1. Result of RFQ design using Eqns. (1-3) with ellipsoidal form factor.

A typical result of this procedure is shown in Fig. 1 for a $140 \mathrm{~mA}, 100 \mathrm{keV}-16 \mathrm{MeV}$ deuteron RFQ ( $8 \mathrm{MeV}$ final energy is considered practical in this case). The EP ratio 
is not well satisfied along the RFQ, although it tends to 1 . The desired individual ratios $\varepsilon_{\varepsilon_{n}} / \varepsilon_{\mathrm{ln}}=\gamma b / a=\sigma^{t} / \sigma^{1}=2$ are also not well satisfied.

This is not too surprising, given the complex longitudinal dynamics. The possible contributions of several effects needed to be investigated. The matching equations (1) and (2) used for accelerator design are actually the equations for a time-invariant periodic transport system. Full envelope equations including the acceleration terms were derived; for RFQs whose parameter changes change slowly enough, as in most extant designs, these terms are small enough to be neglected. Higher-order terms such as would come from rf gaps or quad edges are not a problem in the RFQ.

As especially the longitudinal distribution is changing radically, it is clear that the geometry factor $f f$ is changing in a complicated way, and the usual Sacherer approximation (ellipsoidal distribution, the same in $\mathrm{r}$ and $\mathrm{z}$ directions) is not valid. Additional information is needed, and is obtained from the first design run. A linear correction term for the form factor can be found from the ratio of (total longitudinal beam size/rms longitudinal beam size)/(total transverse beam size/rms transverse beam size). The numerator of this quantity varies from a more cylindrical beam distribution to a parabolic distribution; the denominator is always close to parabolic but does vary systematically.

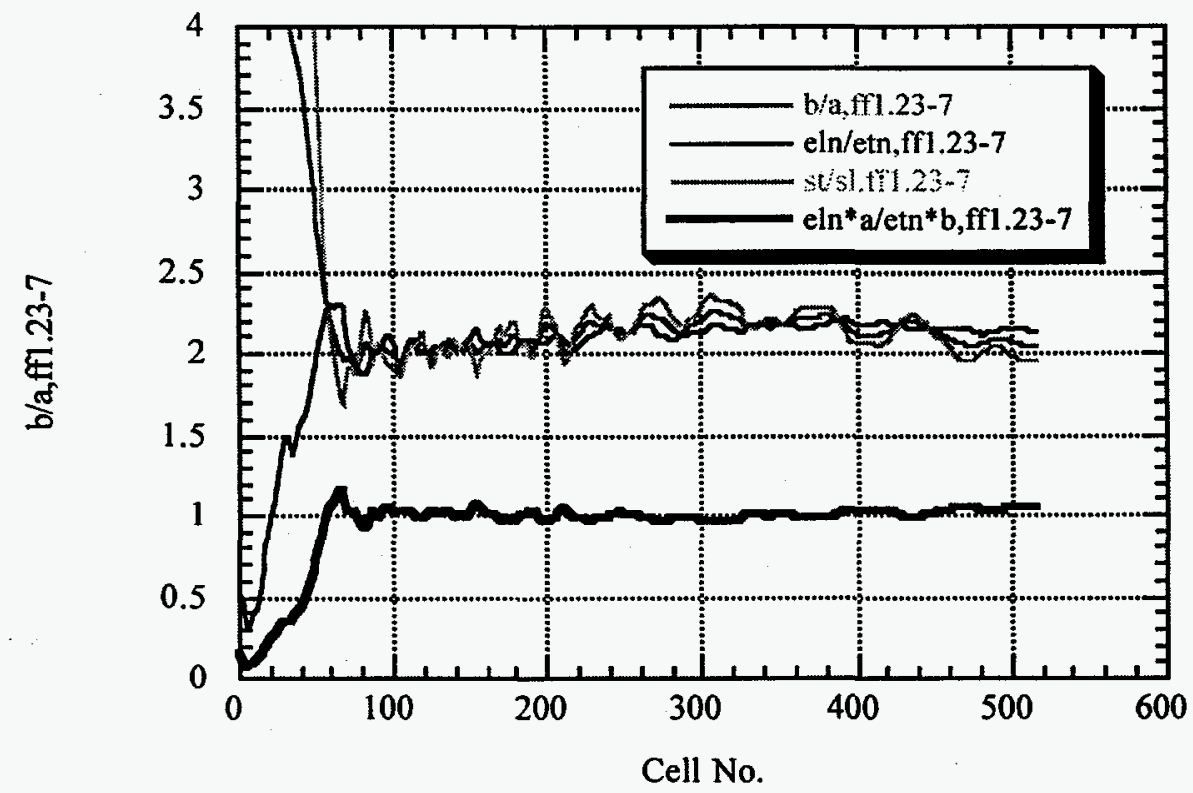

FIGURE 2. Result of RFQ design using Eqns. (1-3) with linear (with Cell No.) correction to the ellipsoidal form factor. 
Application of this correction results in equipartitioning to about $\pm 5 \%$, with individual ratios to about $\pm 10 \%$, as shown in Fig. 2

Other schemes involving the kinetic, potential, space-charge, and nonlinear field energy terms also yield form factor corrections, typically requiring fitting to a 3rdorder polynomial, and also leaving a residual, systematic EP error of about $\pm 5 \%$.

Because the remaining deviation from the design requirement is systematic, and because the goal of the present work is to understand how to achieve the EP condition along the accelerating channel as closely as possible, it is desired to find a higher-order correction. The required form can be seen from numerical experiments, but has not yet been found analytically. The well-known phenomenon that a beam will seek a new distribution in a channel which self-consistently mirrors the external field is clearly happening, and expression of correction terms in this form, as explored by Batygin in this Workshop, is being pursued.

\section{NATURE OF CHAOS}

A final comment on the nature of chaos may be of interest. We have indicated herein and in [9] that resonances play a key role. Space-charge causes wide tune spreads (especially considering the local, instantaneous single-particle tune). Parametric resonances related to the focusing structure are expected to be the most powerful. An infinite number of other resonances exist at all rational number fractions; it is seen that these are used in particle transport. In circular machines, the whole set of reentrant resonances is also introduced. Coherent effects cause shifts in the resonant frequencies as a function of the nonlinearity strength.

Until just a few years ago, the fundamental underpinnings of chaos theory were all based on perturbation theory, and it was known that the perturbation had to be extremely small for the theory to be valid. Yet the indicators, for example the Liapunov coefficients, worked quite well even for strong nonlinearity. This was troubling when reading the literature.

Techniques for geometrically modeling dynamical systems also have a long history. Chaos researchers looked at these and immediately understood that chaos could easily occur on surface manifolds with negative curvature. But they thought chaos could not occur if the curvature was positive. It was realized only a few years ago that this conclusion was wrong. Chaos can also occur if a positively curved manifold has "bumps" on it. And these bumps turn out to be the resonances! A revolution of sorts is now occurring in the literature, toward advanced geometrical methods for surface studies of general Hamiltonians, including time-varying, and deviations from 
the intended surface. The methods are rigorous for strong nonlinearities. It is interesting that resonances, so familiar in accelerators, are the main mechanism, and apparent that these new approaches should be of major relevance to accelerator designers.

\section{REFERENCES}

1 OED/NEA Workshop on "Utilization and Reliability of High Power Proton Accelerators", October 13-15, 1998, Mito, Japan, Proc. to be published by JAERI.

2"IFMIF - International Fusion Materials Irradiation Facility Conceptual Design Activity, Final Report", M. Martone, editor, IFMIF-CDA Team (R.A. Jameson, Accelerator Facility Team Leader), ENEA Frascati Report, RT/ERG/FUS/96/11 (December 1996)..

3 R.A. Jameson, "On Scaling \& Optimization of High Intensity, Low-Beam-Loss RF Linacs for Neutron Source Drivers", AIP Conf. Proc. 279, ISBN 1-56396-191-1, DOE Conf-9206193 (1992) 969-998, Proc. Third Workshop on Advanced Accelerator Concepts, 14-20 June 1992, Port Jefferson, Long Island, NY, LA-UR-92-2474, Los Alamos National Laboratory.

4 R.A. Jameson, "Self-Consistent Beam Halo Studies \& Halo Diagnostic Development in a Continuous Linear Focusing Channel", LA-UR-94-3753, Los Alamos National Laboratory, 9 November 1994. AIP Proceedings of the 1994 Joint US-CERN-Japan International School on Frontiers of Accelerator Technology, Maui, Hawaii, USA, 3-9 November 1994, World Scientific, ISBN 981-02-2537-7, pp.530-560.

5 J.S. O'Connell, et. al., Proc. 1993 Part. Accel. Conf., Washington, DC, May 1993, p. 3657.

6 J.D. Meiss, Physica D74 (1994) 254-267.

7 R. A. Jameson, "Beam-Intensity Limitations in Linear Accelerators," (Invited), Proc. 1981 Particle Accelerator Conf., Washington, DC, March 11-13, 1981, IEEE Trans. Nucl. Sci. 28, p. 2408, June 1981; R. A. Jameson, "Equipartitioning in Linear Accelerators", Proc. of the 1981 Linear Accelerator Conf., Santa Fe, NM, October 19-23, 1981, Los Alamos National Laboratory Report LA9234-C, p. 125, February 1982; I. Hofmann, I. Bozsik, 1981 Linac Conf., LANL, LA-9234-C, pp. 116-119.

${ }^{8}$ I. Hofmann, "Equipartitioning and Halo Due to Anisotropy", Proc. 1997 Part. Accel. Conf.

9 R.A. Jameson, "Beam Losses and Beam Halos in Accelerators for New Energy Sources", HIF Conf., Princeton, NJ, 6-10 Sept. 1995, Princeton, NJ, LA-UR-95-175, Los Alamos National Laboratory, September 1995. Fusion Engineering and Design 32-33 (1996) 149-157. 